\title{
Surgical treatment of traumatic macular hole - Two
}

\section{case reports}

\section{Introduction}

Commotio retinae, diffuse retinal edema, retinal hemorrhage, vitreous hemorrhage, choroidal rupture, photoreceptor lesion, and macular hole are well-established complications of blunt ocular trauma. According to the literature, the frequency of traumatic macular holes is between $1 \%$ and $9 \%{ }^{1}$ The macular hole $(\mathrm{MH})$ may be idiopathic, due to mechanical trauma and even electric current. Authors suggest that, regardless of their etiology, there is an evolutionary sequence with macular edema, cyst formation, and subsequently a macular hole. ${ }^{2}$ Early documentation of cases is an important factor in elucidating the pathophysiological mechanism of MH. Some authors suggest that the traumatic macular hole (TMH) results from the impact of mechanical force transmitted to the macula, leading to rupture of the fovea. ${ }^{3}$ Another theory proposes that a macular cyst caused by blunt trauma may later evolve into a macular hole. ${ }^{4}$ The purpose of this paper is to report two cases of traumatic macular hole treated surgically.

\section{Case reports}

The study protocol was approved by the Institutional Ethics Review Board of the Hospital São Rafael (CAAE: 14929313.4.0000.0048) and conducted by the Retina and Vitreous Service of the same institution.

\section{Case I}

Male patient, 12years old, soccer player, with history of blunt trauma in the right eye with soccer ball 6months ago, attending with low visual acuity (LVA) of this same eye. At ophthalmologic examination showed visual acuity of count finger at 1 meter in the right eye (OD) and 20/20 in the left eye (OS). Biomicroscopy without abnormality and tonometry within normal limits in both eyes. At funduscopy, the patient had a macular hole in the OD and an examination compatible with normality in OS.

Optical coherence tomography (OCT) was performed to confirm the macular hole seen at the fundus examination (Figure 1). The patient was surgically treated with posterior pars plana vitrectomy and peeling of the internal limiting membrane with the aid of the Brilliant Blue dye, followed by tamponade with SF6 gas. It progressed satisfactorily after surgery, with improvement of visual acuity and absence of macular hole at funduscopy. At OCT, it presented macula compatible with post-surgical reorganization, with no evidence of a macular hole. About 1year after the procedure, the patient presented visual acuity of 20/80 with the best correction in OD and no evidence of macular hole to the OCT (Figure 2).

\section{Case 2}

A 43-year-old male patient with a history of trauma with fireworks in the OD 8 months ago, with LVA and "dark spot in vision" since then. Denies previous ophthalmological comorbidities. At ophthalmologic examination: visual acuity of 20/200 in OD and $20 / 20$ in OS. Biomicroscopy showing posterior subcapsular cataract in OD and without abnormalities in OS. 10 and $14 \mathrm{mmHg}$ aplanation tonometry in OD and OS, respectively. Funduscopy presented with macular hole and choroidal rupture in OD and without abnormality in
Volume 9 Issue 2 - 2019

\author{
Adriana do Couto Leitão Guerra,' Paula \\ Cristina Witt, ${ }^{2}$ Bruna Manoela Deschamps, ${ }^{2}$ \\ Ricardo Luz Leitão Guerra,' Otacílio de \\ Oliveira Maia $\mathrm{Jr}^{3}$ \\ 'Department of Ophthalmology, Clinic of Eyes Leitão Guerra, \\ Brazil \\ ${ }^{2}$ Ophthalmology resident at Hospital of Eyes of Paraná, Brazil \\ ${ }^{3}$ Department of Ophthalmology, Hospital São Rafael, Brazil
}

Correspondence: Ricardo Luz Leitão Guerra, Rua Catarina Paraguaçu, n08 - Graça, Salvador (BA), Brazil, CEP, 40.I 50-200, Tel +55 7l 9882288I3, Email ricardo@leitaoguerra.com.br

Received: February 22, 2019 | Published: March 18, 2019

OS (Figure3). OCT examination of the macular region of the patient right eye (Figure 4) confirmed the fundoscopic finding.

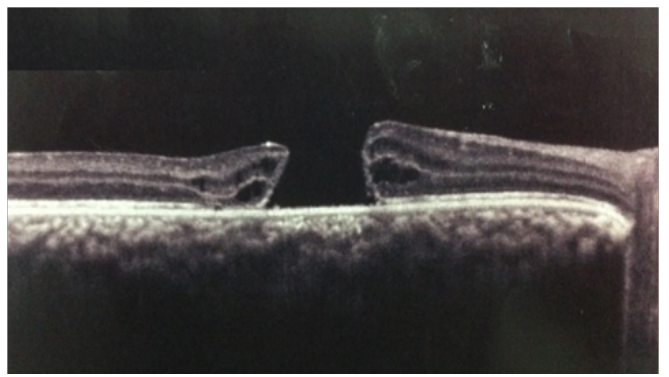

Figure I Preoperative OCT presenting with a macular hole.

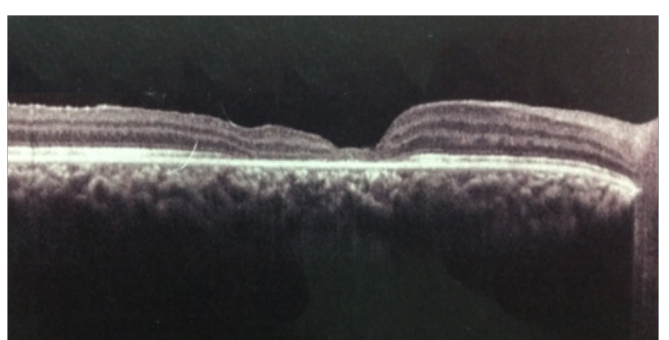

Figure 2 OCT performed in the late postoperative period.

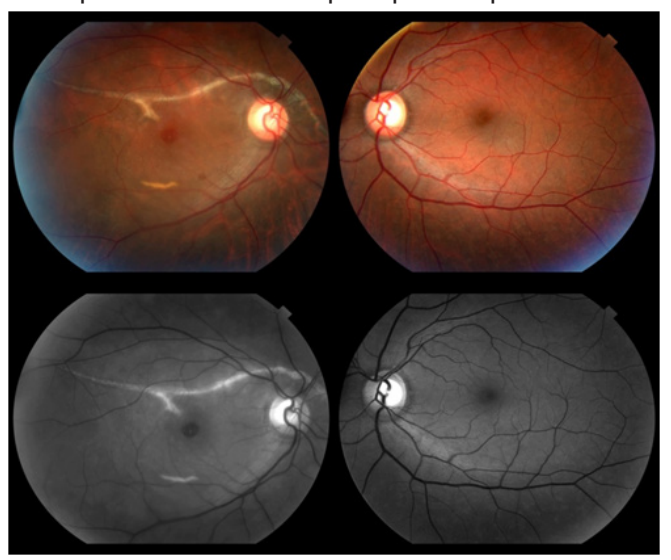

Figure 3 Preoperative fundus photography (color and red-free). Notice the macula hole and a choroidal rupture in the right eye. 


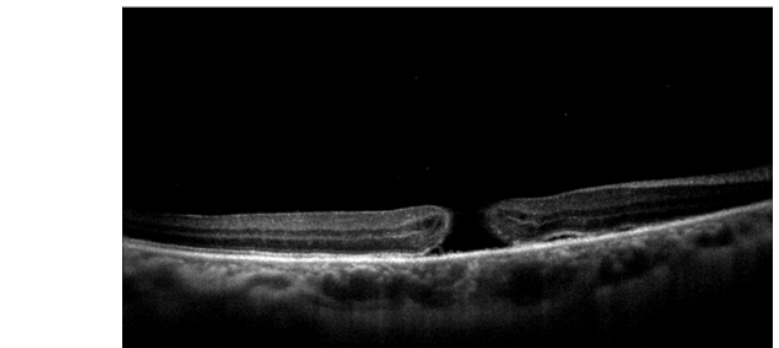

Figure 4 Preoperative OCT presenting with a macular hole.

He underwent phacoemulsification of the lens with intraocular lens implantation in the capsular bag, followed by posterior pars plana vitrectomy and peeling of the inner limiting membrane with the aid of brilliant blue dye and SF6 gas. On the 12th postoperative day the macular hole was closed, seen at funduscopy (Figure 5) and confirmed in the OCT exam (Figure 6). The visual acuity with the best correction on that day was of count finger at 3 meters, without the presence of the central scotoma previously mentioned.

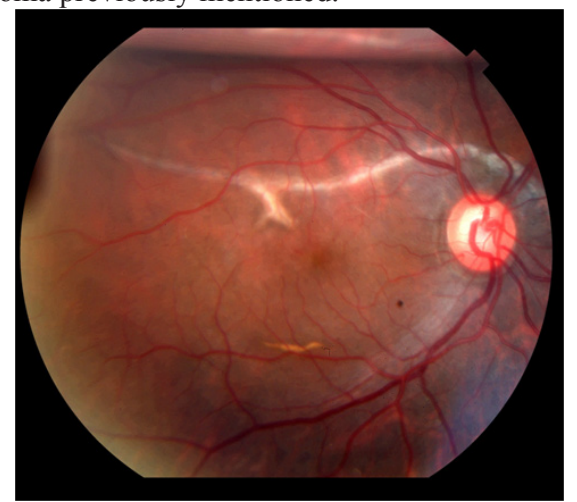

Figure 5 Postoperative fundus photography.

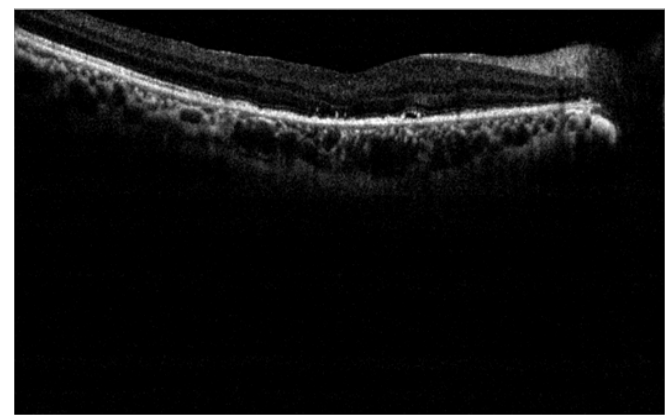

Figure 6 OCT performed in the late postoperative period.

\section{Discussion}

The OCT is a complementary examination of great value, being able to differentiate cases of complete $\mathrm{MH}$ from those with partial involvement of the internal layers of the retina, even with pseudocyst formations and increased macular thickness. In the described cases the OCT examination confirmed the diagnosis and made easy patient orientation and follow-up after surgical treatment.

\section{Conclusion}

Visual results may be good if there is no damage to the pigmented epithelium of the subfoveal retina or Bruch's membrane. ${ }^{5}$ (Spontaneous closure of TMH is not uncommon, with expectant management an option in the first 4 to 6 months after trauma. ${ }^{6,7}$ Vitrectomy seems to be a procedure with high surgical success for TMH closure, with important visual improvement. ${ }^{8-10}$ In the presented cases, the patients already arrived with more than six months of evolution, time in which the spontaneous closure already could have occurred. Therefore surgical treatment was chosen and the result was anatomic and functional improvement of the retina in both cases.

\section{Acknowledgments}

The authors have no conflict of interest to declare. The authors did not receive grant support or research funding. There are no proprietary interests in the materials described in the article. This is an original manuscript. It was presented at a local meeting but wasn't previously submitted for publication. This research was approved by the Institutional Ethics Review Board of the Hospital São Rafael (CAAE: 14929313.4.0000.0048).

\section{Conflicts of interest}

Author declares is conflicts of interest towards this article.

\section{References}

1. Querques G, Barone A, Forte R, et al. Optical coherence tomography and fundus-related perimetry in spontaneous closure of a traumatic macular hole. J Fr Ophtalmol. 2008;31(7):710-713.

2. Haouchine B, Massin P, Tadayoni R, et al. Diagnosis of macular pseudoholes and lamellar macular holes by optical coherence tomography. Am J Ophthalmol. 2004;138(5):732-739.

3. Yanagiya, Akiba J, Takahashi M, et al. Clinical characteristics of traumatic macular holes. Jpn J Ophthalmol. 1996;40(4):544-547.

4. Yamashita T, Uemara A, Uchino E, et al. Spontaneous closure of traumatic macular hole. Am J Ophthalmol. 2002;133(2):230-235.

5. Liu W, Grzybowski A. Surgical treatment of post-traumatic macular holes. J Ophtalmol. 2017;2017:1748135.

6. Menchini U, Virgili G, Giacomelli G, et al. Mechanism of spontaneous closure of traumatic macular hole: OCT study of one case. Retina. 2003;23(1):104-106.

7. Chen YP, Chen TL, Chao AN, et al. Surgical management of traumatic macular hole-related retinal detachment. Am J Ophthalmol. 2005;140(2):331-333.

8. García-Arumí J, Corcostegui B, Cavero L, et al. The role of vitreoretinal surgery in the treatment of posttraumatic macular hole. Retina. 1997; 17(5):372-377.

9. Chen YJ. Vitrectomy and Internal Limiting Membrane Peeling of a Traumatic Macular Hole with Retinal Folds. Case Rep Ophthalmo. 2011;2(1):78-83.

10. Gass JD. Reappraisal of biomicroscopic classification of stages of development of amacular hole. Am J Ophthalmol. 1995;119(6):752-759. 